\title{
射频浌射 $\mathrm{LaNi}_{5}$ 薄膜的吸氢性能研究 ${ }^{*}$
}

\author{
黄 磊 ** 姚伟国 墄震中
}

(中国科学院固体物理研究所,合肥 230031)

\section{关钺词 $\mathrm{LaNi}_{5}$ 薄膜、 $\mathrm{RF}$ 灌射、储量合金}

$\mathrm{LaNi}_{5}$ 是一种研究最活跃的储氢材料 ${ }^{[1]}$, 同时 $\mathrm{LaNi}_{5}$ 薄膜在氢分离、氢电池和氢探测器等 领域内呈现出较有希望的应用前景[2]. 国外对此已经开始广泛的研究, 而国内对储氢薄膜的 研究尚末起步:

储氢合金吸氢可分为氢溶解和生成金属氢化物两个过程. 对于储氢合金薄膜吸氢过程, 国外有学者研究过, 得到不尽相同的结果 ${ }^{[3]}$, 这是一个值得探讨的问题. 另外, 储氢合金吸/放 氢的过程对其中的传导电子将产生影响, 特别是金属氢化物同原合金的电阻率也有差异; 因 而研究储氢薄膜吸/放氢时电阻的变化规律不仅对表征薄膜吸/放氢过程有意义, 而且对薄膜 在氢探测器的应用研究也是重要的.

本文利用镶嵌靶采用射频共浌的办法制备出多晶 $\mathrm{LaNi}_{5}$ 薄膜：通过 $\mathrm{X}$ 射线衍射技术研 究了 $\mathrm{LaNi}_{5}$ 薄膜电解渗氢后相结构的变化, 指出储氢合金薄膜经有效的充氢后可生成 $\beta$ 相的 氢化物. 在四探针法研究 $\mathrm{LaNi}_{5}$ 薄膜吸/放氢时电阻变化的实验中, 观察到了 $\mathrm{LaNi}_{5}$ 薄膜溶解 吸氢和形成金属氢化物所产生的电阻变化.

\section{$1 \mathrm{LaNi}_{5}$ 薄膜的制备}

溅射靶为 $\phi 60 \mathrm{~mm}$ 的四块 $\mathrm{La}$ 和四块 $\mathrm{Ni}$ 采用扇形镶嵌组合而成, 其中 $\mathrm{La}, \mathrm{Ni}$ 的配比要通 过实验确定. 基片选用的是玻璃片和 $\mathrm{Ni}$ 䈃, 浌射时衬底温度约为 $300{ }^{\circ} \mathrm{C}$, 衬底与靶的距离约 为 $40 \mathrm{~mm}$, 贼射气体为氯气, 压强为 $399.996 \times 10^{-3} \mathrm{~Pa}, \mathrm{RF}$ 贼射时的输入功率为 $150 \mathrm{~W}-250 \mathrm{~W}$. 制备的 $\mathrm{LaNi}_{5}$ 薄膜经 $\mathbf{X}$ 光衍射和 $\mathbf{X}$ 光苂光谱分析可以认定是符合化学计量比的多晶 $\mathrm{LaNi}_{5}$ 薄膜.

\section{$2 \mathrm{LaNi}_{5}$ 薄膜吸氢后 $\mathrm{X}$ 射线衍射研究}

以 $\mathrm{Ni}$ 䈃为基片, 在上面溅射沉积 $1.2 \mu \mathrm{m}$ 厚的 $\mathrm{LaNi}_{5}$ 薄膜. 采用电解法充氢 ${ }^{[4]}, \mathrm{LaNi}_{5}$ 薄膜 为阴极, $\mathrm{P}_{\mathrm{t}}$ 网为阳极, 电解液为 $10 \mathrm{ml} \mathrm{H}_{2} \mathrm{SO}_{4}+90 \mathrm{mlH}_{2} \mathrm{O}$, 温度为室温, 电解电流为 $10 \mathrm{~mA}$. 经过 一段时间的充氢, 膜面出现大量气泡, 此时可看作膜中氢量达到饱和. 图 1 给出了 $\mathrm{LaNi}_{5}$ 薄膜 电解渗氢前后的 $\mathrm{X}$ 射线衍射扫描曲线. 从谱图对比可以看出, 电解渗氢对 $\mathrm{LaNi}_{5}$ 薄膜作用明

1993-12-17 收稿, 1994-03-26 收修改稿.

*国家自然科学基金资助项目.

**现在中国科学技术大学材料科学系, 合肥 230026 . 


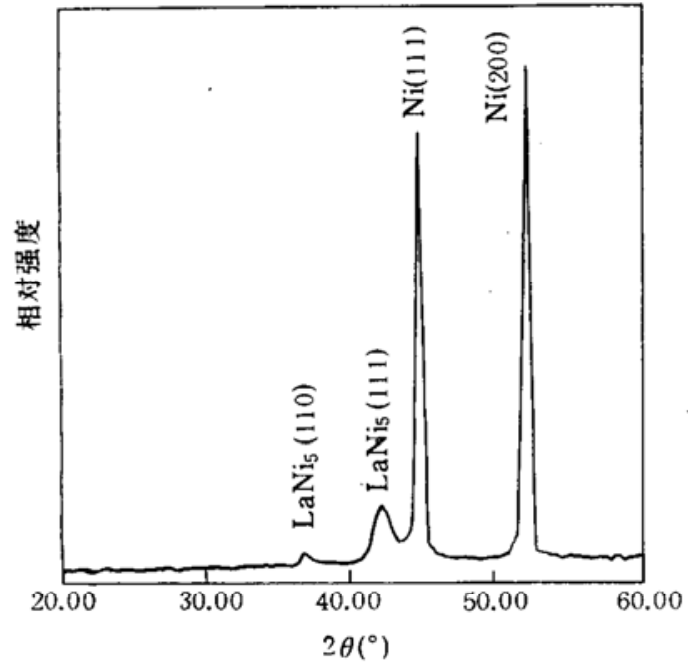

(a)

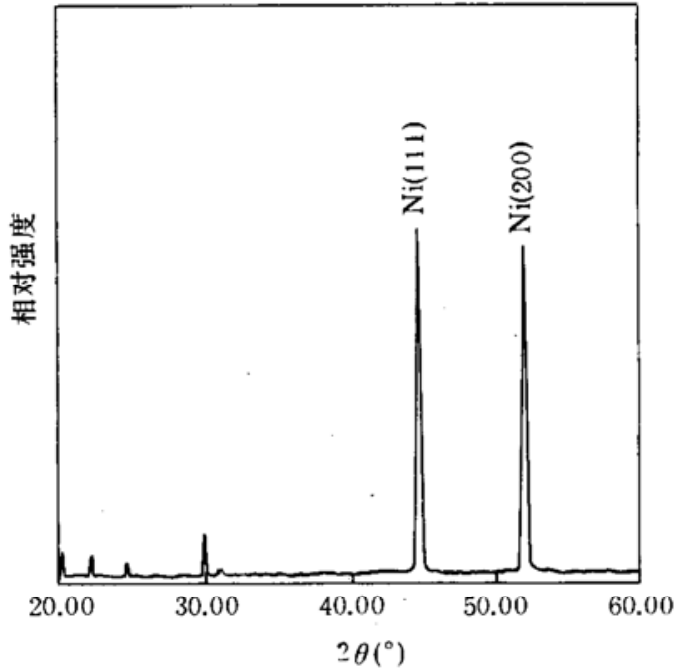

(b)

图 1 沉积在 $\mathrm{Ni}$ 䇴上的 $\mathrm{LaNi}_{5}$ 薄膜的 XRD 谱图

（a）电解渗氢前， (b) 电解渗氢后

显, 原来 $\mathrm{LaNi}_{5}$ 相已转变为 $\mathrm{LaNi}_{5}$ 的金属氢化物相. 这与 $\mathrm{SaKai}^{[3]}$ 报道的结果不同, 他们将 $\mathrm{LaNi}_{5}$ 薄膜充 $40 \mathrm{~atm}$ 的氢压后做 XRD 研究, 只是得到 $\alpha$ 相 $\mathrm{LaNi}_{5}$ 的峰位向低角方向位移并变 宽, 没有金属氢化物生成, 这说明所吸的氢是以溶解的形式存在于 $\mathrm{LaNi}_{5}$ 的晶格间隙. 这一方 面同 $\mathrm{LaNi}_{5}$ 薄膜制备工艺有关, 又同电解渗氢的充氢效率比气相充氢要有效相关.

\section{$3 \mathrm{LaNi}_{5}$ 薄膜吸/放氢时电学性能的研究}

以玻璃片为基片, 在上面沉积 $1.5 \mu \mathrm{m}$ 厚的 $\mathrm{LaNi}_{5}$ 薄膜作为测试试样, 采用电解法充氢, 具 体方法同上所述. 为了比较不同充氢速率的影响, 电解电流分别选取为 $0.1 \mathrm{~mA}, 1 \mathrm{~mA}$ 两种情

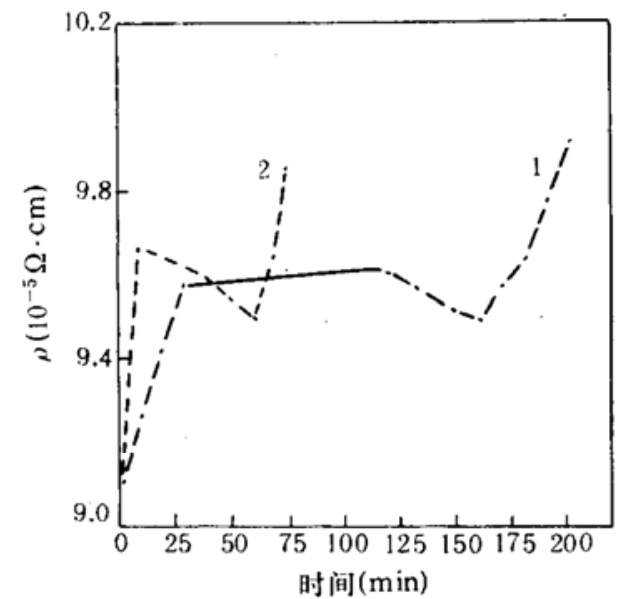

图 $21.5 \mu \mathrm{m}$ 厚的 $\mathrm{LaNi}_{5}$ 薄膜电解充氢时电阻 率随时间的变化

1 为电解电流为 $0.1 \mathrm{~mA}, 2$ 为电解电流为 $1 \mathrm{~mA}$
况. 在电解充氢的同时采用四探针法原位测量其 电阻, 测试电流为 $0.1 \mathrm{~mA}$, 结果见图 2 所示. 从图 中可以看出, 1,2 两条曲线变化规律相同, 在开始 电解渗氢时, 电阻迅速变大, 这对应于氢的溶解过 程, 并且电解电流较大渗氢速度较快, 电阻上升的 也快. 此后, 开始形成良好导电性的氢化物, 曲线 1 由于电解电流较小, 有一个形成氢化物和氢溶 解的平衡过程, 使电阻出现一个较为平稳的区 域. 最后电阻迅速变大是由于 $\mathrm{LaNi}_{5}$ 薄膜吸氢饱 和粉化破裂所致.

研究电解渗氢时 $\mathrm{LaNi}_{5}$ 薄膜电阻的变化, 只 能给出薄膜吸氢时的结果. 为了进一步研究薄膜 吸/放氢时的电学性能, 利用四探针法, 测量了薄 膜在气相情况下吸/放氢时的电阻变化. 测试样 


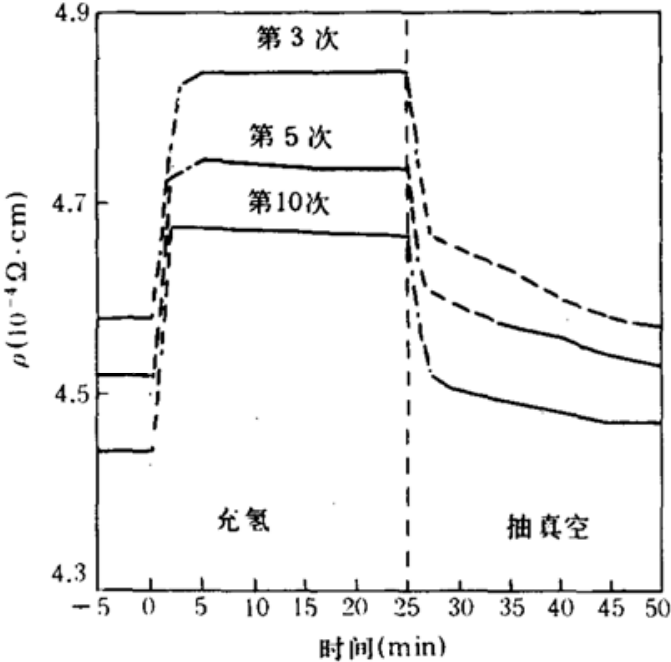

图 3 厚 $0.65 \mu \mathrm{m}$ 的 $\mathrm{LaNi}_{s}$ 薄膜充/放氢 循环时电阻率的变化

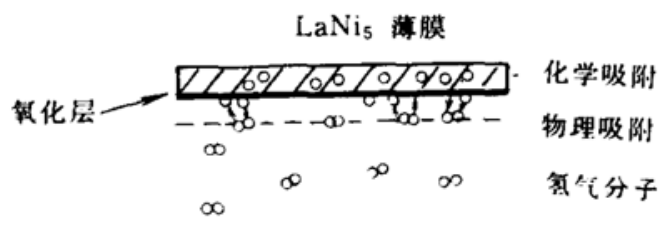

图 4 在氢气气氛下, $\mathrm{LaNi}_{5}$ 薄膜同氢的 作用过程

选用的是以玻璃为基片厚 $0.65 \mu \mathrm{m}$ 的 $\mathrm{LaNi}_{5}$ 薄 膜, 测试温度为 $20^{\circ} \mathrm{C}$, 具体的充/ 放氢循环和 测试方法参见文献[5], 结果见图 3. 图 3 中给 出的充氢结果与电解渗氢的结果相似; 在真空 排气时, 溶解的氢先放出使电阻下降, 而后氢 化物中的氢慢慢释放, 薄膜电阻缓慢下降. 从 图中可以看出第 3 次、 5 次及 10 次循环均有相 似的过程, 只是随着循环的反复进行电阻值减 少. 这可能是经真空排气后, 有少量的氢以某 种形式残留在薄膜中.

\section{4 分 析与 讨 论}

$\mathrm{LaNi}_{5}$ 薄膜在电解渗氢的情况下生成金 属氢化物, 而在 $40 \mathrm{~atm}$ 氢压下并没能生成金 属氢化物 ${ }^{[3]}$, 这可能与它们的充氢方式有关.

在氢气气氛中 $\mathrm{LaNi}_{5}$ 薄膜同氢的作用过 程如图 4 所示. 首先, 氢气分子被合金膜吸附 (物理吸附); 其次, 被吸附的部分氢分子离解 成氢原子(化学吸附); 这时氢原子再通过表 面层向合金膜内扩散, 占据金属原子间的“空 隙”，最后，随氢压增高，溶解氢达到饱和

时, 形成金属氢化物相 $(\mathrm{MH})$.

在电解渗氢时, $\mathrm{LaNi}_{5}$ 薄膜同氢的作用则是按下列过程进行. 首先, 在电极表面通过电化 学反应形成原子氢; 其次, 氢通过表面层向合金膜内部扩散最后形成金属氢化物 $(\mathrm{MH})$.

所以, 在电化学作用下, 氢同合金薄膜生成金属氢化物, 较之在固-气反应生成氢化物要 有效得多; 这主要因为在电化学作用下, 没有氢气的吸附和离解过程, 而这一过程在气相吸 氢中决定着薄膜的吸氢效率, 特别是 $\mathrm{LaNi}_{5}$ 薄膜表面覆盖一层 $\mathrm{La}_{2} \mathrm{O}_{3}$ 层 $(10 \mathrm{~nm}-30 \mathrm{~nm})$, 如不经 过活化, 是不能很快吸附氢分子.

$\mathrm{LaNi}_{5}$ 薄膜吸/放氢影响其电阻变化主要因为在吸氢过程中, 溶解的氢占据金属原子间的 “空隙”, 束缚邻近的金属中的传导电子, 使导带中传导电子减少, 电阻升高; 此后, 随着薄膜中 氢压增高, 溶解的氢与合金形成良导电性的氢化物, 所以电阻下降. 真空排气后, 首先没有形 成氢化物溶解的氢向膜面扩散并变为氢分子逸出, 被其束缚的传导电子释放出来, 使导电带 电子增多, 电阻下降; 之后, 随薄膜中氢分压降低导致氢化物中的氢脱离氢化物, 电阻又有上 升趋势. 由此可见在吸/放氢过程中薄膜电阻的变化反映着吸/放氢的过程. 即吸氢时先溶 解吸氢然后形成金属氢化物; 放氢时, 先释放溶解的氢再释放金属氢化物中的氢. 这同 $\mathrm{LaNi}_{5}$ 合金吸/放氢时的 P-C-T 图对应. 


\section{5 结 论}

$\mathrm{LaNi}_{5}$ 薄膜的吸氢特性同其块体材料相比有自己的特点. 首先薄膜的吸氢量低于块体材 料, 其次 $\mathrm{LaNi}_{5}$ 薄膜在吸/放氢循环中有抗粉化的性能 ${ }^{[3]}$. 吸氢性能有差异, 但薄膜同体材 吸/放氢的过程和机理是相同的, $\mathrm{LaNi}_{5}$ 薄膜并不只是溶解吸氢如经有效的充氢可以生成金属 氢化物. 造成薄膜吸氢量较小与薄膜的衬底效应有关, 由于褯底的存在限制了 $\mathrm{LaNi}_{5}$ 吸氢时 的晶格膨胀, 从而限制了吸氢量, 这也是 $\mathrm{LaNi}_{5}$ 薄膜抗粉化的主要原因.

$\mathrm{LaNi}_{5}$ 薄膜吸/放氢时电阻的变化, 间接反映着薄膜吸/放氢的过程, 进一步探讨 $\mathrm{LaNi}_{5}$ 薄 膜吸氢时电阻的变化率以及测量的灵敏度对薄膜在氢探测器应用上是有意义的.

\section{参 考 文 献}

[1] Cohen, R. L., Wernick, J. H., Science, 1981, 214: 1081.

[2] Sakaguchi, H., Seri, H., Adachi, G., J. Phy. Chem., 1990, 94:5313.

[3] Sakai, T., Lshikcawa, H. et al., J. Electrochem., Soc., 1991, 138(4): 908.

[4] 胡小军、邬秋林、满一军,物理学报, 1992, 41(10): 1575.

[5] Adachi, G., Sakaguchi, H., Niki, K. et al., J. Less Common Met., 1985, 108: $107-114$. 